\title{
Systemic cytokines and GlycA discriminate disease status and predict corticosteroid response in HTLV- 1 -associated neuroinflammation
}

\section{Tatiane Assone}

University of São Paulo Brazil/Institute of Tropical Medicine of São Paulo

\section{Soraya Maria Menezes}

Rega Institute for Medical Research, KU Leuven

\section{Fernanda Toledo}

Rega Institute for Medical Research, KU Leuven

\section{Victor Angelo Folgosi}

University of São Paulo Brazil/Institute of Tropical Medicine of São Paulo

\section{Gabriela Silva Prates}

University of São Paulo Brazil/Institute of Tropical Medicine of São Paulo

\section{Tim Dierckx}

Rega Institute for Medical Research, KU Leuven

\section{Marcos Braz}

Rega Institute for Medical Research, KU Leuven

\section{Jerusa Smid}

University of São Paulo Brazil/Institute of Tropical Medicine of São Paulo Michel E. Haziot

University of São Paulo Brazil/Institute of Tropical Medicine of São Paulo Rosa M N Marcusso

University of São Paulo Brazil/Institute of Tropical Medicine of São Paulo

Flávia E. Dahy

University of São Paulo Brazil/Institute of Tropical Medicine of São Paulo

\section{Roberta Bruhn}

Vitalant research Institute

\section{Edward L. Murphy}

University of California San Francisco

\section{Augusto César Penalva Oliveira}

University of São Paulo Brazil/Institute of Tropical Medicine of São Paulo

\section{Dirk Daelemans}

Rega Institute for Medical Research, KU Leuven 
Rega Institute for Medical Research, KU Leuven

Jorge Casseb

University of São Paulo Brazil/Institute of Tropical Medicine of São Paulo

Johan Van Weyenbergh ( $\sim$ johan.vanweyenbergh@kuleuven.be )

Rega Institute for Medical Research, KU Leuven

\section{Research Article}

Keywords: HTLV-1, HAM/TSP, cytokines, inflammation, corticosteroids

Posted Date: February 17th, 2022

DOI: https://doi.org/10.21203/rs.3.rs-1359301/v1

License: (c) (1) This work is licensed under a Creative Commons Attribution 4.0 International License. Read Full License 


\section{Abstract}

Background: HTLV-1-Associated Myelopathy/Tropical Spastic Paraparesis (HAM/TSP) is an incapacitating neuroinflammatory disorder for which no disease-modifying therapy is available, but corticosteroids provide some clinical benefit. Although HAM/TSP pathogenesis is not fully elucidated, older age, female sex and higher proviral load are established risk factors. We investigated systemic cytokines and a novel chronic inflammatory marker, GlycA, as possible biomarkers of immunopathogenesis and therapeutic response in HAM/TSP, and examined their interaction with established risk factors.

Patients and Methods: We recruited 110 People living with HTLV-1 (PLHTLV-1, 67 asymptomatic individuals and $43 \mathrm{HAM} / \mathrm{TSP}$ patients) with a total of 946 person-years of clinical follow-up. Plasma cytokine levels (IL-2, IL-4, IL-6, IL-10, IL-17A, IFN- $y$, TNF) and GlycA were quantified by Cytometric Bead Array and ${ }^{1} \mathrm{NMR}$, respectively. Cytokine signaling and prednisolone response were validated in an independent cohort by nCounter digital transcriptomics. We used multivariable regression, machine learning algorithms and Bayesian network learning for biomarker identification.

Results: We found that systemic IL- 6 was positively correlated with both age $(r=0.50, p<0.001)$ and GlycA $(r=0.45, p=0.00049)$ in asymptomatics, revealing an 'inflammaging" signature which was absent in HAM/TSP. GlycA levels were higher in women $(p=0.0069)$, but cytokine levels did not differ between the sexes. IFN- $\gamma(p=0.007)$ and IL-17A $(p=0.0001)$ levels were increased in untreated HAM/TSP Multivariable logistic regression identified IL-17A and proviral load as independent determinants of clinical status, resulting in modest accuracy of predicting HAM/TSP status (64.1\%), while a machine learning-derived decision tree classified HAM/TSP patients with $90.7 \%$ accuracy. Pre-treatment GlycA and TNF levels significantly predicted clinical worsening (measured by Osame Motor Disability Scale), independent of proviral load. In addition, a poor prednisolone response was significantly correlated with higher posttreatment IFN-y levels. Likewise, a transcriptomic IFN signaling score, significantly correlated to previously proposed HAM/TSP biomarkers (CASP5/CXCL10/FCGR1A/STAT1), was efficiently blunted by in vitro prednisolone treatment of PBMC from PLHTLV-1 and incident HAM/TSP.

Conclusions: An age-related increase in systemic IL-6/GlycA levels reveals inflammaging in PLHTLV-1, in the absence of neurological disease. IFN- $y$ and IL-17A are biomarkers of untreated HAM/TSP, while pretreatment GlycA and TNF predict therapeutic response to prednisolone pulse therapy, paving the way for a precision medicine approach in HAM/TSP.

\section{Introduction}

Human T-cell Lymphotropic Virus type-1 (HTLV-1) is unique as it is both oncogenic $[1,2]$ and capable of triggering HTLV-1-Associated Myelopathy/Tropical Spastic Paraparesis (HAM/TSP) and other inflammatory diseases [3-5]. Worldwide, 10 million people are estimated to be living with HTLV-1 
(PLHTLV-1) [3], of which 1-2\% develop HAM/TSP, an incapacitating, progressive neuroinflammatory disorder $[4,5]$.

Currently, no disease-modifying therapy is available for HAM/TSP but corticosteroids and other immunomodulators (IFN-a, methotrexate, cyclosporin) provide some clinical benefit [4-6]. Moreover, we recently demonstrated HAM/TSP is an independent predictor for early mortality among PLHTLV-1 [7]. As such, validated biomarkers to predict and/or monitor disease progression for PLHTLV-1 and therapeutic outcome in HAM/TSP patients are direly needed [8].

Acute inflammation is routinely measured by systemic cytokines, while glycoprotein acetylation (GlycA) represents a novel marker for chronic inflammation, which reliably predicts long-term outcomes of inflammatory and infectious diseases in patient cohorts or large prospective population studies [9-12]. Up to now, GlycA has not been explored in HTLV-1 infection or HAM/TSP. Lifelong chronic infection with other latent viruses (CMV and HIV) causes long-term activation of the immune system over time, contributing to inflammaging [13]. Although extensively described in people living with HIV-1 [14.15], data on inflammaging are lacking in PLHTLV-1. Therefore, we investigated pro- and anti-inflammatory cytokines and GlycA as possible biomarkers of inflammaging, immunopathogenesis and therapeutic response in HAM/TSP, using unique samples from a large cohort of PLHTLV-1 with an exceptionally long clinical follow-up (range 2-20 years).

\section{Patients And Methods}

\section{Cohort characteristics, patient recruitment and sampling strategy}

From and ongoing open cohort study researching the natural history of HTLV-1 infection [7], we selected all first available plasma samples (closest to recruitment into the cohort) from patients with definite HAM/TSP before treatment, as well as age- and gender-matched first available samples (closest to recruitment into the cohort) of PLHTLV-1 who were neurologically asymptomatic (AS) during clinical follow-up (study period: August 1997 to December 2019). All volunteers underwent serological screening for HTLV-1 at the "Emilio Ribas" Institute of Infectious Diseases, using GOLD ELISA HTLV-1/ 2 (Diasorin, UK), followed by confirmation with Western Blot (MP Diagnostics, HTLV Blot 2.4®) and in-house nested PCR [16]. Paired follow-up samples were obtained after methylprednisolone pulse therapy for 38 HAM/TSP patients. Blood samples were collected in $\mathrm{K}_{3}$-EDTA $(0.054 \mathrm{ml} /$ tube), plasma was separated by centrifugation (15 min, $2500 \mathrm{rpm}$ ) and PBMC were purified by Ficoll density gradient centrifugation (GE Healthcare Life, USA ). Cells were washed with saline solution, the cell number was adjusted to $10^{6}$ cells, followed by storage (as "dry pellet") at $-80^{\circ} \mathrm{C}$. DNA was extracted using a commercial kit (Illustra Tissue and Cells Genomic Prep Mini Spin kit, Fairfield, CA) according to the manufacturer's instructions, and stored at $-80^{\circ} \mathrm{C}$.

\section{Quantification of HTLV-1 proviral load (PVL)}


HTLV-1 proviral load was quantified by real-time PCR, using primers and probes targeting the HTLV-1 pol gene, with the human albumin gene as internal reference, as described previously [16]. All samples were analyzed in duplicate, and results expressed as HTLV- 1 DNA copies $/ 10^{6}$ PBMCs.

\section{Neurological evaluation and HAM/TSP diagnosis}

PLHTLV-1 were classified in two groups according to their neurological status: 67 asymptomatic HTLV-1infected individuals and 43 HAM/TSP patients, of which 38 provided paired samples after treatment with methylprednisolone (1 $\mathrm{g}$ intravenously, every 45 days). HAM/TSP diagnostic criteria was based on recommendations from an international consortium [17]. Clinical evaluation and a standardized screening neurological examination were performed by a board-certified neurologist, blinded for HTLV-1 clinical status for all subjects. For clinical follow-up, the Osame Motor Disability Scale was used, ranging from 0 (no walking or running disabilities) to 13 (cannot even move toes).

\section{Plasma cytokine levels}

Concentrations of plasma (undiluted) cytokines were measured using the CBA (Cytometric Bead Array, BD Biosciences) Human Th1/Th2/Th17 Cytokine Kit, including Interleukin-2 (IL-2), Interleukin-4 (IL-4), Interleukin-6 (IL-6), Interleukin-10 (IL-10), Tumor Necrosis Factor (TNF), Interferon-ץ (IFN-ץ), and Interleukin-17A (IL-17A), in accordance with the manufacturer's instructions.

\section{GlycA (Glycoprotein Acetyl) quantification in plasma}

GlycA concentration was quantified using the Nightingale Health Ltd. high-throughput metabolomics platform (Helsinki, Finland), as previously described [9]. Briefly, a ${ }^{1} \mathrm{H}-\mathrm{NMR}$ spectrum is taken from $350 \mu \mathrm{l}$ of plasma, with the area under the peak measured at approximately $2 \mathrm{ppm}$ quantifying signal originating from $\mathrm{N}$-acetyl sugar groups present on acute phase glycoproteins ( $\mathrm{a}-1$-acid glycoprotein, $\mathrm{a}-1$-antitrypsin, a-1-antichymotryspin, haptoglobin, transferrin).

\section{Data collection and Quality Control}

Data entry in the electronic database RedCap [18] was performed by two administrative assistants, and subsequently checked by the first and last author.

\section{Ethical Issues}

The Ethical Board of "Instituto de Infectologia Emilio Ribas", Sao Paulo-Brazil, approved the protocol (Number 07688818.2.1001.0061). Signed informed consent was obtained from all participants prior to study inclusion.

\section{Digital transcriptomics and biological pathway analysis}

Digital transcriptomic analysis (nCounter, Nanostring Technologies) and biological pathway analysis of in vitro prednisolone response was performed as previously described [19, 20], using the Myeloid/Innate Immunity Panel, consisting of $>750$ host genes, as well as customized HTLV-1 Hbz and Tax probes. 
Specific cytokine signaling scores were calculated as z-scores of the geometric mean of a set of curated transcripts (Suppl. Table 2) for each cytokine or cytokine family (IL-10, IFN, IL-17, IL4/IL-13, IL-2 family). PBMCs were obtained from an independent, previously characterized cohort of PLHTLV-1 (4 asymptomatic, 4 HAM/TSP patients), as well as age-, gender- and ethnicity-matched healthy controls ( $n=$ 4) from the HOST study [21].

\section{Statistical analysis, Machine Learning and Bayesian Network analysis}

Statistical analysis was performed using XLStat and GraphPad Prism version 9, San Diego, CA). Logistic regression and non-parametric statistical tests (Mann-Whitney, Wilcoxon tests, Spearman correlation) were used, except for Maximal Osame Motor Disability Score (which followed normal distribution, ANOVA), with Bonferroni correction for multiple comparisons as indicated in the text. For prednisolone response in vitro, one sample t test was used to compare groups to the mean value of incident HAM/TSP, since numbers ( 4 in each group) were too small to apply generalized linear mixed models. Machine learning algorithms (attribute selection, J48 and PART decision trees) were applied using Weka (version 3.8.4). Bayesian network analysis was performed as previously described [22].

\section{Results}

Age and gender differentially affect cytokines and GlycA in people living with HTLV-1, independent of proviral load

As shown in Table 1, HAM/TSP patients were age- and gender-matched to HTLV-1-infected controls without neurological symptoms (asymptomatics, AS), while proviral load was increased in HAM/TSP, as expected $[4,5]$. Since age and gender are major determinants of HAM/TSP pathogenesis, as disease onset usually occurs after several decades and women are more affected [4,5], we investigated if cytokines or GlycA were linked to these demographics, in the absence or presence of neuroinflammation. Biological sex did not influence systemic cytokine levels (data not shown), whereas both IL-6 (Spearman's $r=0.36, p=0.00018)$ and IL-10 $(r=0.22, p=0.021)$ were positively correlated with age in PLHTLV-1

(Fig. 1A-1D). Surprisingly, this age-dependent cytokine increase was specific to $A S(r=0.50, p<0.0001$, Fig. 1B-1E), and absent in HAM/TSP (Fig. 1C-1F). In contrast, chronic inflammation marker GlycA did not correlate with age $(p=0.12)$, but was higher in females $(p=0.0069$, Fig. $1 G)$. Among all cytokines, only IL6 was significantly correlated to GlycA in AS (Fig. $1 \mathrm{H}, p=0.00049, r=0.45)$ but not HAM/TSP patients ( $p$ $=0.16$ ), revealing an "inflammaging" signature which was surprisingly limited to PLHTLV-1 without neuroinflammatory disease. Correlations of IL-6 with age and with GlycA remained significant after Bonferroni correction for multiple testing, while IL-10 correlation with age was not. Of note, none of the cytokines nor GlycA were significantly correlated to proviral load (Fig. 1 I and not shown), but a tendency was observed for IFN-y (Fig. 1I, $r=0.20, p=0.054)$. 
Table 1

Principal characteristics of study participants (AS vs. HAM/TSP)

\begin{tabular}{|c|c|c|c|}
\hline Variables & Asymptomatics & HAM/TSP & P value ${ }^{a}$ \\
\hline Gender n (\%) & & & 0.24 \\
\hline Male & $20(30.8)$ & $14(31.1)$ & \\
\hline Female & $47(68.2)$ & $29(68.9)$ & \\
\hline \multicolumn{4}{|c|}{ Age (years) in entry of cohort ${ }^{b}$} \\
\hline Median $( \pm S D)$ & $51(13)$ & $48(13)$ & 0.40 \\
\hline \multicolumn{4}{|c|}{ Time Follow-up (years) } \\
\hline Median $( \pm S D)$ & $8.6(5.7)$ & $8.6(3.7)$ & 0.37 \\
\hline Range (min-max) & $1.5-20.8$ & $1.5-18.6$ & \\
\hline \multicolumn{4}{|c|}{ HTLV-1 Proviral Load ${ }^{c}$} \\
\hline Median & 3,845 & $28,184(14,464-53,864)$ & 0.0007 \\
\hline$(95 \% \mathrm{Cl})$ & $(839-18,953)$ & & \\
\hline \multicolumn{4}{|c|}{ aStatistical tests: Chi-square for categorical variables, Mann-Whitney test for numerical variables } \\
\hline \multicolumn{4}{|c|}{${ }^{\mathrm{b}}$ Age missing for $1 \mathrm{HAM} / \mathrm{TSP}$} \\
\hline${ }^{\mathrm{c}} \mathrm{HTLV}-1$ DNA cop & roviral load miss & for $10 \mathrm{AS}$ and $4 \mathrm{HAM} / \mathrm{TSP}$ & \\
\hline
\end{tabular}

\section{Pro-inflammatory cytokines IFN-y and IL-17A are biomarkers of untreated HAM/TSP}

When comparing cytokine levels between clinical groups, we observed a significant increase in IFN- $\mathrm{p}(\mathrm{p}=$ $0.007)$ and IL-17A ( $p=0.0001)$ in HAM/TSP patients, as compared to AS, while other cytokines and GlycA did not differ (Fig. 2A). Using logistic regression (detailed in Suppl. Table 1), we found that IL-17A and proviral load were independently associated with clinical status, consistent with a weak correlation between IFN-y and proviral load (Fig. 1I). However, logistic regression resulted in low classification accuracy for HAM/TSP patients, as only 25/39 (64.1\%) were correctly classified, in contrast to 49/57 (86.0\%) of correctly predicted AS (ROC AUC 0.85 ). Therefore, we used machine learning algorithms to improve classification, which revealed a decision tree (Fig. 2B) classifying 39/43 HAM/TSP and 58/67 AS, respectively with $90.7 \%$ and $86.6 \%$ accuracy (ROC AUC 0.87 ). Among the first branches in this decision tree are IL-17A and IL-10, confirming previous findings in a UK cohort [23].

Bayesian networks are graphical models that are widely used in systems biology to depict genes/proteins as nodes and connections between genes as edges, based on both linear and non-linear associations 
between the nodes. Thus, network connections in Bayesian networks have been demonstrated to accurately capture the functional and mechanistic relationship of genes/proteins that can inform on disease mechanisms and help define composite sets of molecular markers [24-26]. Therefore, we used Bayesian network learning to identify direct vs. indirect associations between cytokines, GlycA, clinical and demographic data, similar to previous analysis in HTLV-1-associated leukemia [22]. As shown in Fig. 2C, only IL-17A was directly connected to clinical status, while all other cytokines were 'upstream' of IL-17A. This Bayesian network revealed a direct link between GlycA and IL-6, whereas the observed correlation between age and IL-6 (Fig. 1A-B) appears dependent on TNF and IFN- - , the latter directly influencing GlycA. IFN- $\gamma$ was found upstream of all other cytokines and, consequently, of disease status, which underscores the previously identified IFN gene signature in HAM/TSP [27]. Of interest, in this unsupervised model, proviral load was not significantly associated with the other parameters and hence absent from the network.

\section{Systemic cytokines and GlycA are biomarkers of corticosteroid therapeutic response in HAM/TSP}

Next, we investigated if cytokines and GlycA might be candidate biomarkers for therapeutic response in HAM/TSP patients. In this cohort, all eligible patients were uniformly treated with intravenous methylprednisolone pulse therapy, which allowed unbiased comparisons before and after treatment. Patients with $>1$ year pulse therapy follow-up were classified as responders $(n=13)$ and non-responders $(n=25)$, based on changes in Osame Motor Disability Score (decrease or $\leq 1$ : responders, increase $>1$ : non-responders). All patients significantly decreased IL-17A levels after treatment (Fig. 3A, $p=0.013$ ), while strong variability but no directionality was observed for any other cytokine, nor for GlycA (Fig. 3A). However, prednisolone-responders displayed lower IFN- $y$ levels after treatment ( $p=0.010$, Fig. 3B). In addition, pre-treatment TNF levels were significantly associated with therapeutic outcome (Fig. 3B, $p=$ 0.011). Likewise, pre-treatment GlycA levels were able to predict therapeutic response, (measured by quantitative changes in Osame Motor Disability Score), either by itself (Fig. 3C, left panel) or as a combined TNF/GlycA score (Fig. 3C, right panel). Multivariable linear regression (Suppl. Table 2) confirmed both TNF and GlycA as independent predictors of disability progression. This finding, in addition to GlycA's relative stability over time [9-11], underscores the potential of GlycA as a novel and clinically useful biomarker in HAM/TSP.

\section{Transcriptomic validation of cytokine signatures in PLHTLV- 1 and incident HAM/TSP}

To confirm and extend our findings of cytokine signatures at the protein level (Fig. 1-4), we used digital transcriptomics (nCounter) to provide broader mechanistic insight into the downstream cytokine signaling pathways $[19,20]$ mediating HAM/TSP disease progression, and the effect of prednisolone treatment in vitro. From a well-characterized US cohort of PLHTLV-1 [21], we selected 4 AS and 4 HAM/TSP patients (including the only two incident HAM/TSP cases from the entire HTLV-1 cohort), and age-, gender- and ethnicity-matched healthy controls $(n=4)$. First, we confirm that exacerbated IFN 
signaling is a hallmark of HAM/TSP disease status [27], being significantly higher in incident HAM/TSP (Fig. 4A), as compared to healthy controls (five-fold, $p=0.022$ ) and AS (four-fold $p=0.0085$ ), when quantified ex vivo by nCounter. Likewise, ex vivo IL-17 signaling score was also increased in incident HAM/TSP (Fig. 4B), as compared to healthy controls (two-fold, $p=0.044$ ) and AS (1.5-fold, $p=0.14$ ). Similar to our findings in HAM/TSP patients from the Brazilian cohort, IFN signaling ( 3.5 -fold, $p=0.0096)$ and IL-17 signaling score (two-fold, $p=0.089$ ) were downregulated in the US cohort at (4-year) follow-up. However, no uniform HAM/TSP treatment protocol exists for the US HOST cohort, and the number of patients was too small to correlate cytokine signaling in vitro to therapeutic response or disability score.

Nevertheless, IFN signaling (measured by nCounter) was effectively and homogeneously down-regulated in all clinical groups by in vitro prednisolone treatment (Fig. 4C), which was further confirmed by decreased expression of the IFN-y-regulated MHC Class II antigen presentation pathway (Fig. 4C). In contrast, in vitro prednisolone treatment did not have a uniform effect on IL-10 signaling, IL4/IL-13 signaling, IL-17 signaling or IL-2 cytokine family signaling (data not shown) but was strongly variable among the four clinical groups. Finally, we investigated if nCounter IFN signaling score was correlated to previously identified HAM/TSP transcriptomic biomarkers (CASP5, FCGR1A, STAT1 identified in [27]) and CXCL 10, proposed by several groups $[4,5,8,41]$ as a sensitive biomarker for HAM/TSP disease status and corticosteroid response. As shown in Fig. 4D, ex vivo CASP5, CXCL10, FCGR1A and STAT1 transcript levels were significantly (all $\mathrm{p}<0.05$ with Bonferroni correction) correlated to IFN signaling score. In contrast, HTLV-1 transcripts Hbz and Tax were not significantly correlated to IFN signaling score (Fig. 4D), suggesting that different cytokine signatures in PLHLTV-1 and HAM/TSP patients are not directly triggered by retroviral transcription.

\section{Discussion}

In this observational study, we selected 110 biobanked plasma samples, corresponding to all first available samples, i.e. closest to the date of entry in a large open cohort of PLHTLV-1, corresponding to a total of 946 person-years of clinical follow-up, including 43 HAM/TSP patients and 67 asymptomatic PLHTLV-1. Using these unique samples, we demonstrated systemic cytokines and GlycA as candidate biomarkers of inflammaging, immunopathogenesis and therapeutic response in HAM/TSP.

Inflammaging has been extensively documented in people living with HIV-1 (14.15), but this is the first report of inflammaging in PLHTLV-1, characterized by an age-dependent increase in pro-inflammatory cytokine IL-6, which was positively correlated to chronic inflammation marker GlycA (Fig. 2). Among proinflammatory cytokines, IL-6 uniquely predicts global functional decline in aging [28] and inflammaging in a systematic review and meta-analysis [29]. Although IL-10 was weakly correlated with age in asymptomatics, the IL6/IL10 ratio, representing a higher pro-inflammatory state, significantly increases with age $( \pm 50 \% / 15 y e a r s, p=0.014)$, and also corroborates the increased mortality rate we observed in this cohort [7]. Since IL6/IL10 ratio has been demonstrated as a sensitive biomarker of COVID-19 outcome [30], aging PLHTLV-1 might be at increased risk of developing severe or critical COVID-19. 
Although this hypothesis was also supported by in silico findings [31], it remains to be confirmed in large observational studies, which are challenging in neglected diseases [32].

Regarding disease status, we have used complimentary analytical approaches, namely multivariable regression, machine learning-derived decision trees and Bayesian network learning. While multivariable logistic regression identified IL-17A and proviral load as independent predictors of HAM/TSP disease status, Bayesian network analysis enables visualization of the co-dependencies between cytokines in PLHTLV-1, and their associations with GlycA and disease status. Thus, we found that IL-17A appears intrinsically related to disease status in all three analytical models (regression, decision tree and Bayesian network). However, IFN-y appears "upstream" of all other cytokines in the Bayesian network, which corroborates previous transcriptomic findings [27]. This study confirms the findings of Kagdi et al. for IFN-y and IL-17A as biomarkers of untreated HAM/TSP [23]. In spite of our larger cohort (67 AS, 43 HAM/TSP), we did not observe increased IL-2 nor IL-10 in HAM/TSP, in contrast to Kagdi et al. (17 AS, 28 HAM/TSP). However, our decision tree (Fig. 2B) identified by Machine Learning is quite similar to the decision tree proposed by Kagdi et al. to classify AS, HAM/TSP and ATL patients based upon IL-10 and IL-17 levels. Exacerbated IFN-y production has been consistently demonstrated by numerous groups, either ex vivo (in serum/plasma) or in vitro (in supernatants of PBMCs cultured for 1-4 days), as a hallmark of HAM/TSP $[4,5,23,33-38]$. Other pro- and anti-inflammatory cytokines (IL-2, IL-4, IL-6, IL-10, IL-17A, TNF) have yielded strongly diverging results between different groups regarding their up- or downregulation in HAM/TSP vs. AS [23,33-40]. This is most likely due to smaller cohort sizes, in addition to the choice of plasma/serum vs. cell culture supernatants or intracellular flow cytometry. Additionally, differences in age, gender, genetics as well as the inclusion of treated HAM/TSP patients in some cohorts might also explain the observed discrepancies. Indeed, we found that IFN- $y$ and IL-17A are differentially impacted by corticosteroid pulse therapy: post-treatment IFN- $\gamma$ levels are low in responders, while IL-17A levels decrease uniformly for all patients. Current clinical guidelines for HAM/TSP suggest that early HAM/TSP patients might benefit most from corticosteroid therapy [6], which is also supported the recent (and first placebo-controlled) randomized clinical trial for corticosteroid therapy in HAM/TSP (HAMLET-P [41]).

In addition to IL-17A, we found proviral load is an independent biomarker of untreated disease in HAM/TSP patients, consenting with the literature [4-7]. However, proviral load did not predict incident HAM/TSP cases in three out of four published Brazilian cohort studies [42-45]. Of those, only Tanajura et al. demonstrated proviral load as a significant predictor of neurological symptoms, but not definite HAM/TSP, during clinical follow-up [43]. Similar to Yamauchi et al. [8], we found that proviral load is not a biomarker for therapeutic response in HAM/TSP. However, we identified, for the first time, TNF and GlycA as independent predictors of clinical worsening, as measured by increased Osame Motor Disability Scale. To put this prediction into a patient-centered clinical context, the median Osame Motor Disability Scale of 4 in the low TNF/GlycA group corresponds to "needs a handrail when climbing stairs", whereas the median of 8 in the high TNF/GlycA group corresponds to "can walk 1-5m with bilateral support". Notably, the high TNF/GlycA group also comprised the only fatal case among 43 HAM/TSP patients, with death related to HAM/TSP as described in our previous study [7]. 
Notable strengths and limitations of this study merit further detail. First, this study has a relatively large sample size, to our knowledge the largest yet with regards to plasma cytokines as candidate biomarkers in PLHTLV-1 and HAM/TSP. Second, complete neurological evaluations of PLHTLV-1 and uniform treatment strategy for HAM/TSP patients are major strengths, as well as a remarkably long follow-up (median 8.6 year in Brazil cohort, > 14 year in US cohort). A major limitation is the low incidence in the US cohort (2/2100 person-years), thus limiting our statistical power for replication. Other limitations include the lack of simultaneous protein and RNA quantification in both cohorts (due to sample availability), as well as potential selection biases regarding patient recruitment and loss to follow-up [7], which are inherent to cohort studies in neglected diseases.

\section{Conclusion}

We found that the untreated disease status in HAM/TSP patients, as compared to age-and gendermatched asymptomatic PLHTLV-1, is characterized by increased systemic IFN- $\gamma$ and IL-17A. From a clinical point of view, plasma GlycA, IL-6, TNF and IFN-y are promising candidate biomarkers for immunomonitoring of inflammaging in PLHTLV-1, and of disease progression and corticosteroid therapeutic response in HAM/TSP patients, respectively. In addition, we provide predictive regression models and decision trees for prospective testing in clinical trials or independent cohort studies.

\section{Abbreviations}

AS: asymptomatics

HAM/TSP: HTLV-1-Associated Myelopathy/Tropical Spastic Paraparesis

HIV: Human Immunodeficiency Virus

HTLV: Human T-cell Leukemia virus

IFN: interferon

IL: interleukin

PLHTLV-1: People Living with HTLV-1

TNF: Tumor Necrosis Factor

\section{Declarations}

\section{- Consent for publication:}

All authors have seen and approved the manuscript and agree to its publication. 
All authors declare no conflict of interest.

\section{- Availability of data and materials:}

All data contained in the manuscript are available from the corresponding authors.

\section{- Funding}

This research was funded by FAPESP grant numbers 2017/08320-5, 2018/07239-2 and 2016/03025-2 (scholarship to TA); CNPq (Scholarship to JC), FFM (support to JC), FWO (grant G0A0621N to JVW) andKU Leuven ("Vaast Leysen Leerstoel voor Infectieziekten in Ontwikkelingslanden" to JVW). Specimen acquisition for the HTLV Outcomes Study was funded by a grant (R01-HL-62235) from the U.S. National Heart, Lung and Blood Institute (NHLBI).

\section{- Authors' contributions}

TA, SMM, MB, TD and JVW performed experiments and analyzed data. FTG, VA, GSP, JS, MEH, RMNM, FED, RB, EM, ACPO and JC provided patient samples and clinical data. JV and DD participated in cytokine and statistical analysis. TA, JC and JVW wrote the main manuscript text and JVW prepared figures 1-4. All authors reviewed the manuscript.

\section{Acknowledgments:}

The authors would like to thank all interns of the IIER Neurology program, and all persons living with HTLV-1 for their participation.

\section{References}

1. Tagaya Y, Gallo RC. The Exceptional Oncogenicity of HTLV-1. Front Microbiol. 2017;8:1425.

2. Poiesz BJ, Ruscetti FW, Reitz MS, Kalyanaraman VS, Gallo RC. Isolation of a new type C retrovirus (HTLV) in primary uncultured cells of a patient with Sézary T-cell leukaemia. Nature. 1981;294:26871.

3. Gessain A, Cassar O. Epidemiological Aspects and World Distribution of HTLV-1 Infection. Front Microbiol. 2012;3:388.

4. Bangham CRM, Araujo A, Yamano Y, Taylor GP. HTLV-1-associated myelopathy/tropical spastic paraparesis. Nat Rev Dis Primer. 2015;1:15012.

5. Nozuma S, Jacobson S. Neuroimmunology of Human T-Lymphotropic Virus Type 1-Associated Myelopathy/Tropical Spastic Paraparesis. Front Microbiol. 2019;10:885.

6. Araujo A, Charles R.M. Bangham CRM, Casseb J, Gotuzzo E, MD, Jacobson S, Martin F, Oliveira AP, MD, Puccioni-Sohler M, Graham GP, Yamano Y, on behalf of the International Retrovirology Association. Management of HAM/TSP Systematic review and consensus-based recommendations 2019. Neurology: Clinical Practice. 0:1-8. 
7. Marcusso RMN, Van Weyenbergh J, de Moura JVL, Dahy FE, de Moura Brasil Matos A, Haziot MEJ, et al. Dichotomy in Fatal Outcomes in a Large Cohort of People Living with HTLV-1 in São Paulo, Brazil. Pathogens. 2019;9.

8. Yamauchi J, Araya N, Yagishita N, Sato T, Yamano Y. An update on human T-cell leukemia virus type I (HTLV-1)-associated myelopathy/tropical spastic paraparesis (HAM/TSP) focusing on clinical and laboratory biomarkers. Pharmacol Ther. 2021 Feb;218:107669.

9. Dierckx T, Verstockt B, Vermeire S, Van Weyenbergh J. GlycA, a Nuclear Magnetic Resonance Spectroscopy Measure for Protein Glycosylation, is a Viable Biomarker for Disease Activity in IBD. J Crohns Colitis. 2019;13:389-94.

10. Ritchie SC, Würtz P, Nath AP, Abraham G, Havulinna AS, Fearnley LG, et al. The Biomarker GlycA Is Associated with Chronic Inflammation and Predicts Long-Term Risk of Severe Infection. Cell Syst. 2015;1:293-301.

11. Dierckx T, Chiche L, Daniel L, Lauwerys B, Weyenbergh JV, Jourde-Chiche N. Serum GlycA Level is Elevated in Active Systemic Lupus Erythematosus and Correlates to Disease Activity and Lupus Nephritis Severity. J Clin Med. 2020 Mar 31;9(4):970.

12. Lawler PR, Akinkuolie AO, Chandler PD, Moorthy MV, Vandenburgh MJ, Schaumberg DA, Lee IM, Glynn RJ, Ridker PM, Buring JE, Mora S. Circulating N-Linked Glycoprotein Acetyls and Longitudinal Mortality Risk. Circ Res. 2016 Apr 1;118(7):1106-15.

13. Franceschi C, Bonafe M, Valensin S, Olivieri F, De Luca M, Ottaviani E, et al. Inflamm-aging. An evolutionary perspective on immunosenescence. Ann N Y Acad Sci. 2000;908:244-54.

14. 14.. Babu H, Ambikan AT, Gabriel EE, Svensson Akusjärvi S, Palaniappan AN, Sundaraj V, Mupanni NR, Sperk M, Cheedarla N, Sridhar R, Tripathy SP, Nowak P, Hanna LE, Neogi U. Systemic Inflammation and the Increased Risk of Inflamm-Aging and Age-Associated Diseases in People Living With HIV on Long Term Suppressive Antiretroviral Therapy. Front Immunol. 2019 Aug 27;10:1965.

15. Sieg SF, Shive CL, Panigrahi S, Freeman ML. Probing the Interface of HIV and Inflammaging. Curr HIV/AIDS Rep. 2021 Jun;18(3):198-210.

16. Montanheiro PA, Montanheito PA, Oliveira ACP de, Posada-Vergara MP, Milagres AC, Tauil C, et al. Human T-cell lymphotropic virus type I (HTLV-I) proviral DNA viral load among asymptomatic patients and patients with HTLV-I-associated myelopathy/tropical spastic paraparesis. Braz J Med Biol Res. 2005;38:1643-7.

17. De Castro-Costa CM, Araújo AQC, Barreto MM, Takayanagui OM, Sohler MP, da Silva ELM, et al. Proposal for diagnostic criteria of tropical spastic paraparesis/HTLV-I-associated myelopathy (TSP/HAM). AIDS Res Hum Retroviruses. 2006;22:931-5.

18. Harris PA, Taylor R, Thielke R, Payne J, Gonzalez N, Conde JG. Research electronic data capture (REDCap)-a metadata-driven methodology and workflow process for providing translational research informatics support. J Biomed Inform. 2009;42:377-81. 
19. Fukutani KF, Nascimento-Carvalho CM, Bouzas ML, Oliveira JR, Barral A, Dierckx T, et al. In situ Immune Signatures and Microbial Load at the Nasopharyngeal Interface in Children with Acute Respiratory Infection. Front Microbiol. 2018;9:2475.

20. Moens B, Pannecouque C, López G, Talledo M, Gotuzzo E, Khouri R, Bittencourt A, Farré L, GalvãoCastro B, Vandamme AM, Van Weyenbergh J. Simultaneous RNA quantification of human and retroviral genomes reveals intact interferon signaling in HTLV-1-infected CD4 + T cell lines. Virol J. 2012 Aug 23;9:171.

21. Kwaan N, Lee T-H, Chafets DM, Nass C, Newman B, Smith J, et al. Long-term variations in human T lymphotropic virus (HTLV)-I and HTLV-II proviral loads and association with clinical data. J Infect Dis. 2006;194:1557-64.

22. Khouri R, Silva-Santos G, Dierckx T, Menezes SM, Decanine D, Theys K, et al. A genetic IFN/STAT1/FAS axis determines CD4 T stem cell memory levels and apoptosis in healthy controls and Adult T-cell Leukemia patients. Oncoimmunology. 2018;7:e1426423.

23. Kagdi H, Demontis MA, Ramos JC, Taylor GP. Switching and loss of cellular cytokine producing capacity characterize in vivo viral infection and malignant transformation in human T- lymphotropic virus type 1 infection. PLoS Pathog. 2018 Oct 17];14.

24. Y. Chen, J. Zhu, P.Y. Lum, X. Yang, S. Pinto, D.J. Macneil, C. Zhang, J. Lamb, S. Edwards, S.K. Sieberts, A. Leonardson, L.W. Castellini, S. Wang, M.F. Champy, B. Zhang, V. Emilsson, S. Doss, A. Ghazalpour, S. Horvath, T.A. Drake, A.J. Lusis, E.E. Schadt. Variations in DNA elucidate molecular networks that cause disease Nature, 452 (2008), pp. 429-435

25. B. Zhang, C. Gaiteri, L.G. Bodea, Z. Wang, J. Mcelwee, A.A. Podtelezhnikov, C. Zhang, T. Xie, L. Tran, R. Dobrin, E. Fluder, B. Clurman, S. Melquist, M. Narayanan, C. Suver, H. Shah, M. Mahajan, T. Gillis, J. Mysore, M.E. Macdonald, J.R. Lamb, D.A. Bennett, C. Molony, D.J. Stone, V. Gudnason, A.J. Myers, E.E. Schadt, H. Neumann, J. Zhu, V. Emilsson. Integrated systems approach identifies genetic nodes and networks in late-onset Alzheimer's disease. Cell, 153 (2013), pp. 707-720.

26. Meng Q, Zhuang Y, Ying Z, Agrawal R, Yang X, Gomez-Pinilla F. Traumatic Brain Injury Induces Genome-Wide Transcriptomic, Methylomic, and Network Perturbations in Brain and Blood Predicting Neurological Disorders. EBioMedicine. 2017 Feb;16:184-194. doi: 10.1016/j.ebiom.2017.01.046.

27. Tattermusch S, Skinner JA, Chaussabel D, Banchereau J, Berry MP, McNab FW, et al. Systems biology approaches reveal a specific interferon-inducible signature in HTLV-1 associated myelopathy. PLoS Pathog. 2012;8:e1002480.

28. Adriaensen W, Matheï C, Vaes B, van Pottelbergh G, Wallemacq P, Degryse J-M. Interleukin-6 as a firstrated serum inflammatory marker to predict mortality and hospitalization in the oldest old: A regression and CART approach in the BELFRAIL study. Exp Gerontol. 2015;69:53-61.

29. Marcos-Pérez D, Sánchez-Flores M, Proietti S, Bonassi S, Costa S, Teixeira JP, et al. Association of inflammatory mediators with frailty status in older adults: results from a systematic review and meta-analysis. GeroScience. 2020;42:1451-73. 
30. McElvaney OJ, Hobbs BD, Qiao D, McElvaney OF, Moll M, McEvoy NL, Clarke J, O'Connor E, Walsh S, Cho MH, Curley GF, McElvaney NG. A linear prognostic score based on the ratio of interleukin- 6 to interleukin-10 predicts outcomes in COVID-19. EBioMedicine. 2020 Nov;61:103026.

31. Ghosh N, Saha I, Sharma N. Interactome of human and SARS-CoV-2 proteins to identify human hub proteins associated with comorbidities. Comput Biol Med. 2021 Nov;138:104889.

32. Galvão-Castro B, Rios Grassi MF, Nunes A, Galvão-Barroso AK, Galvão-Castro AV, Lírio M, Ribeiro A, de Faria Junqueira T, Silva AL, Cerqueira M, Rangel SL, Araujo THA, Boa-Sorte N, Dourado I, Castro-Lima $\mathrm{H}$, Soliani MLC. Challenges in establishing telehealth care during the COVID-19 pandemic in a neglected HTLV-1-infected population in northeastern Brazil. PLoS Negl Trop Dis. 2020 Dec 31;14(12):e0008922.

33. Yamano Y, Araya N, Sato T, Utsunomiya A, Azakami K, Hasegawa D, et al. Abnormally high levels of virus-infected IFN-gamma + CCR4 + CD $4+C D 25+T$ cells in a retrovirus-associated neuroinflammatory disorder. PloS One. 2009;4:e6517.

34. Starling AL, Coelho-Dos-Reis JG, Peruhype-Magalhães V, Pascoal-Xavier MA, Gonçalves DU, Béla SR, Lambertucci JR, Labanca L, Souza Pereira SR, Teixeira-Carvalho A, Ribas JG, Trindade BC, Faccioli $\mathrm{LH}$, Carneiro-Proietti AB, Martins-Filho OA. Immunological signature of the different clinical stages of the HTLV-1 infection: establishing serum biomarkers for HTLV-1-associated disease morbidity. Biomarkers. 2015;20(6-7):502-12.

35. Ando H, Sato T, Tomaru U, Yoshida M, Utsunomiya A, Yamauchi J, Araya N, Yagishita N, Coler-Reilly A, Shimizu Y, Yudoh K, Hasegawa Y, Nishioka K, Nakajima T, Jacobson S, Yamano Y. Positive feedback loop via astrocytes causes chronic inflammation in virus-associated myelopathy. Brain. 2013 Sep;136(Pt 9):2876 - 87.24.

36. Furuya T, Nakamura T, Fujimoto T, Nakane S, Kambara C, Shirabe S, Hamasaki S, Motomura M, Eguchi K. Elevated levels of interleukin-12 and interferon-gamma in patients with human $T$ lymphotropic virus type l-associated myelopathy. J Neuroimmunol. 1999 Mar 1;95(1-2):185-9.

37. Montanheiro PA, Penalva de Oliveira AC, Smid J, Fukumori LM, Olah I, da S Duarte AJ, Casseb J. The elevated interferon gamma production is an important immunological marker in HAM/TSP pathogenesis. Scand J Immunol. 2009 Oct;70(4):403-7.

38. Leal FE, Ndhlovu LC, Hasenkrug AM, Bruno FR, Carvalho KI, Wynn-Williams H, Neto WK, Sanabani SS, Segurado AC, Nixon DF, Kallas EG. Expansion in CD39âo CD4âo immunoregulatory t cells and rarity of Th17 cells in HTLV-1 infected patients is associated with neurological complications. PLoS Negl Trop Dis. 2013;7(2):e2028.

39. Romanelli LCF, Miranda DM, Carneiro-Proietti ABF, Mamede M, Vasconcelos HMM, Martins ML, Ferreira ASD, Rosa DVF, Paula JJ, Romano-Silva MA, Nicolato R. Spinal cord hypometabolism associated with infection by human T-cell lymphotropic virus type 1(HTLV-1). PLoS Negl Trop Dis. 2018 Aug 27;12(8):e0006720.

40. Santos SB, Oliveira P, Luna T, Souza A, Nascimento M, Siqueira I, Tanajura D, Muniz AL, Glesby MJ, Carvalho EM. Immunological and viral features in patients with overactive bladder associated with 
human T-cell lymphotropic virus type 1 infection. J Med Virol. 2012 Nov;84(11):1809-17.

41. Yamauchi J, Tanabe K, Sato T, Nakagawa M, Matsuura E, Tsuboi Y, Tamaki K, Sakima H, Ishihara S, Ohta Y, Matsumoto N, Kono K, Yagishita N, Araya N, Takahashi K, Kunitomo Y, Nagasaka M, ColerReilly A, Hasegawa Y, Araujo A, Jacobson S, Grassi MFR, Galvão-Castro B, Bland M, Taylor GP, Martin F, Yamano Y. Efficacy of Corticosteroid Therapy for HTLV-1-Associated Myelopathy: A Randomized Controlled Trial (HAMLET-P). Viruses. 2022 Jan 12;14(1):136.

42. Romanelli LC, Caramelli P, Martins ML, Gonçalves DU, Proietti FA, Ribas JG, Araújo MG, CarneiroProietti AB. Incidence of human T cell lymphotropic virus type 1-associated myelopathy/tropical spastic paraparesis in a long-term prospective cohort study of initially asymptomatic individuals in Brazil. AIDS Res Hum Retroviruses. 2013 Sep;29(9):1199-202.

43. Tanajura D, Castro N, Oliveira P, Neto A, Muniz A, Carvalho NB, Orge G, Santos S, Glesby MJ, Carvalho EM. Neurological Manifestations in Human T-Cell Lymphotropic Virus Type 1 (HTLV-1)-Infected Individuals Without HTLV-1-Associated Myelopathy/Tropical Spastic Paraparesis: A Longitudinal Cohort Study. Clin Infect Dis. 2015 Jul 1;61(1):49-56.

44. Neurologic, clinical, and immunologic features in a cohort of HTLV-1 carriers with high proviral loads. Ferraz SN, Costa GF, Carneiro Neto JA, Hebert T, de Oliveira CJV, Guerra M, Oliveira LMA, Carvalho EM. J Neurovirol. 2020 Aug;26(4):520-529.

45. Martins ML, Guimarães JC, Ribas JG, Romanelli LC, de Freitas Carneiro-Proietti AB. Long-term followup of HTLV-1 proviral load in asymptomatic carriers and in incident cases of HAM/TSP: what is its relevance as a prognostic marker for neurologic disease? J Neurovirol. 2017 Feb;23(1):125-133.

\section{Figures}



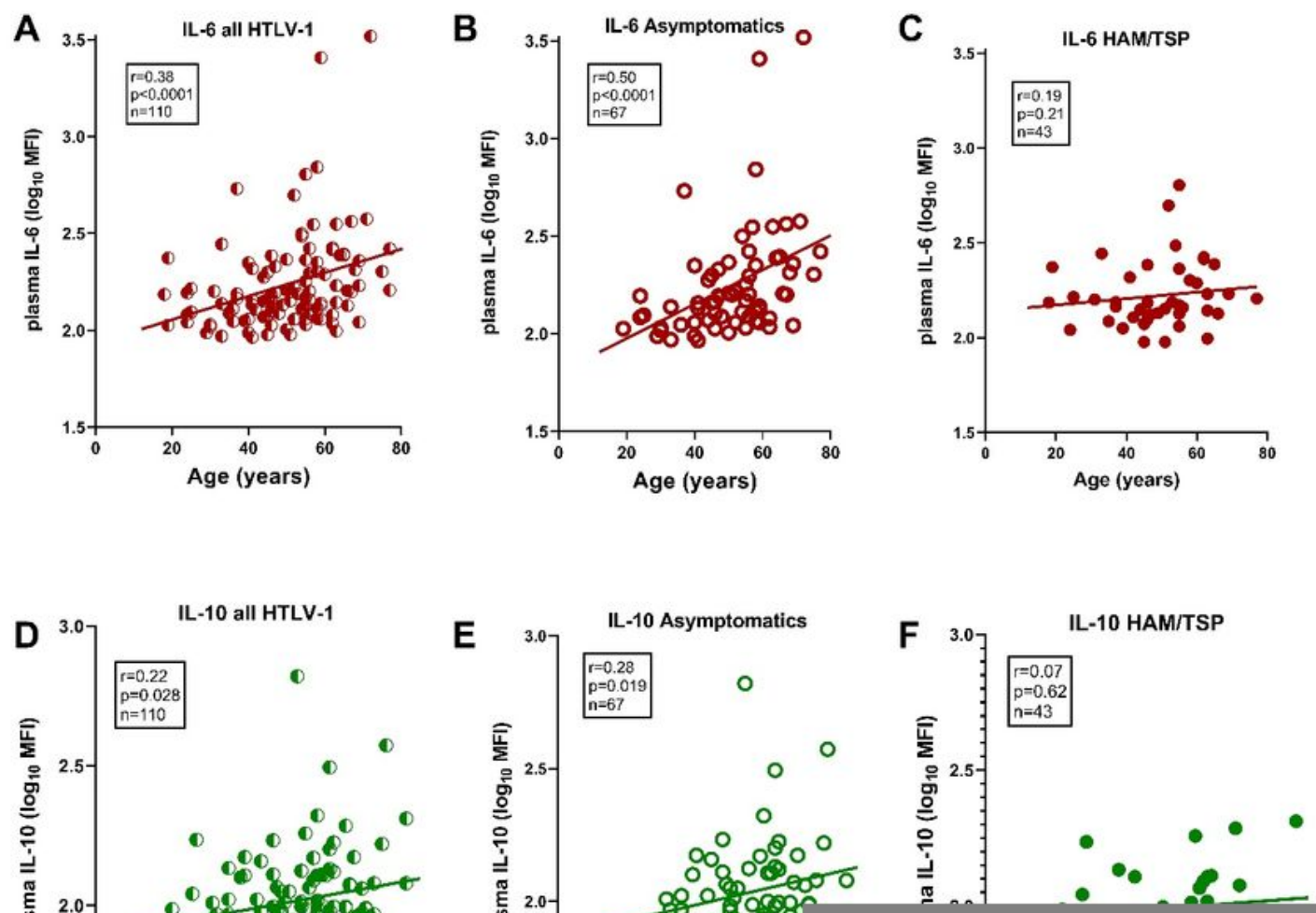

Figure 1

Age and gender differentially affect cytokines and chronic inflammation marker GlycA in people living with HTLV-1, independent of proviral load. (A) IL-6 levels are significantly correlated to age at sampling in all PLHTLV-1, which is driven by the strong correlation in asymptomatic individuals (AS) (B), which is absent in HAM/TSP patients (C). (D) IL-10 levels are significantly correlated to age at sampling in all PLHTLV-1, which is driven by the strong correlation in AS (E), which is absent in HAM/TSP patients (F). 
(G) GlycA levels are significantly higher in female PLHTLV-1 ( $p=0.0069$, Mann-Whitney test). (H) GlycA levels are positively correlated to IL-6 levels in AS only $(p=0.00049, r=0.45)$. (I) Proviral load is not significantly correlated to IL-6 $(\rho=0.07, p=0.45)$ or GlycA levels $(\rho=-0.06, p=0.72)$ in PLHTLV- 1 , while a tendency is observed for IFN- $\gamma(\rho=0.20, p=0.054)$. Correlation is determined using Spearman's method, uncorrected $p$-values are reported, significant correlations of IL-6 with age and with GlycA were robust to correction for multiple testing (Bonferroni $\mathrm{p}<0.05$ ).

\section{Figure 2}

\section{HAM/TSP disease status is characterized by increased IFN- $\gamma$ and IL-17A}

(A) Among all cytokines tested, only IFN- $y(p=0.0054)$ and IL-17A $(p<0.0001)$ levels in HAM/TSP patients $(\mathrm{n}=43)$ were significantly different (Mann-Whitney test), as compared to asymptomatic individuals (AS, $n=67$ ), both associations were robust to correction for multiple testing (Bonferroni $p<0.05$ ). (B) Machine learning-derived decision tree discriminating AS from HAM/TSP patients (J48 pruned tree) with $90 \%$ accuracy (97 out of 110 PLHTLV-1 correctly classified, of which 61/67 AS and 38/43 HAM/TSP patients, ROC AUC 0.87, Kappa statistic 0.79). (C) Bayesian Network representing the strongest associations between cytokines, GlycA, clinical and demographic data (strength of arcs as defined previously [19]: dark blue 100x, light blue 10x). Proviral load was not significantly associated with the other parameters and hence not shown.

\section{Figure 3}

Cytokines and GlycA predict therapeutic success vs. failure of methylprednisolone pulse therapy in HAM/TSP patients.

(A) Among all plasma cytokines measured in HAM/TSP patients, only IL-17A significantly decreases after pulse therapy with intravenous methylprednisolone (Wilcoxon test $p=0.003$, Bonferroni correction $p<0.05$ ).

(B) Pre-treatment TNF (left panel) and post-treatment IFN-y (right panel) are correlated with the magnitude of clinical worsening after prednisolone pulse therapy (Spearman correlation). (C) Pretreatment GlycA levels are correlated with the magnitude of clinical worsening after methylprednisolone pulse therapy (Spearman correlation, left panel). A higher TNF/GlycA score $\left(0.025^{\star} \mathrm{TNF}+9.18 * \mathrm{GlycA}\right.$ 7.28) predicts worse Osame Motor Disability Score after methylprednisolone pulse therapy (right panel, ANOVA with Bonferroni post-test, ${ }^{*} \mathrm{p}=0.010$ ). 

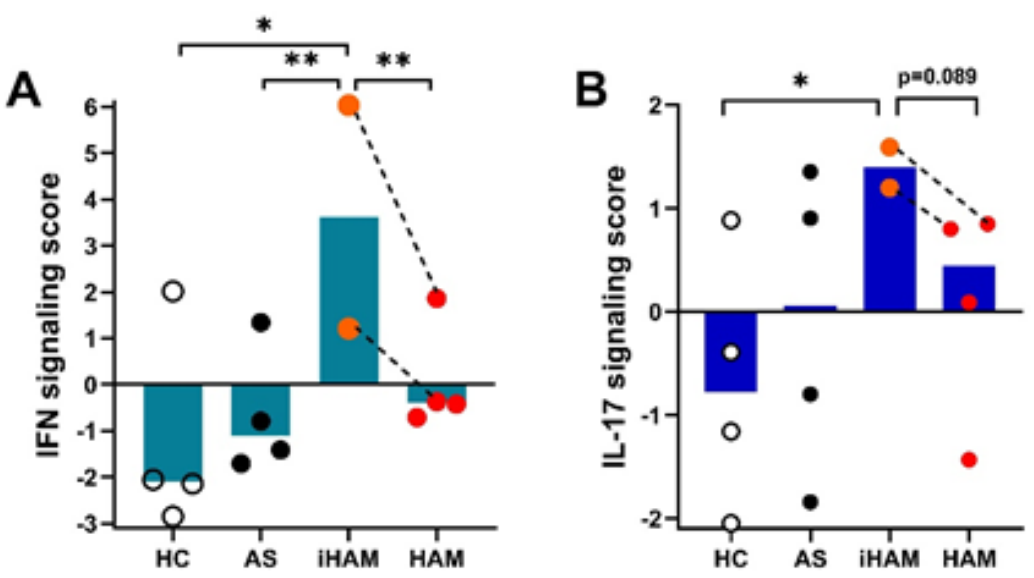

C
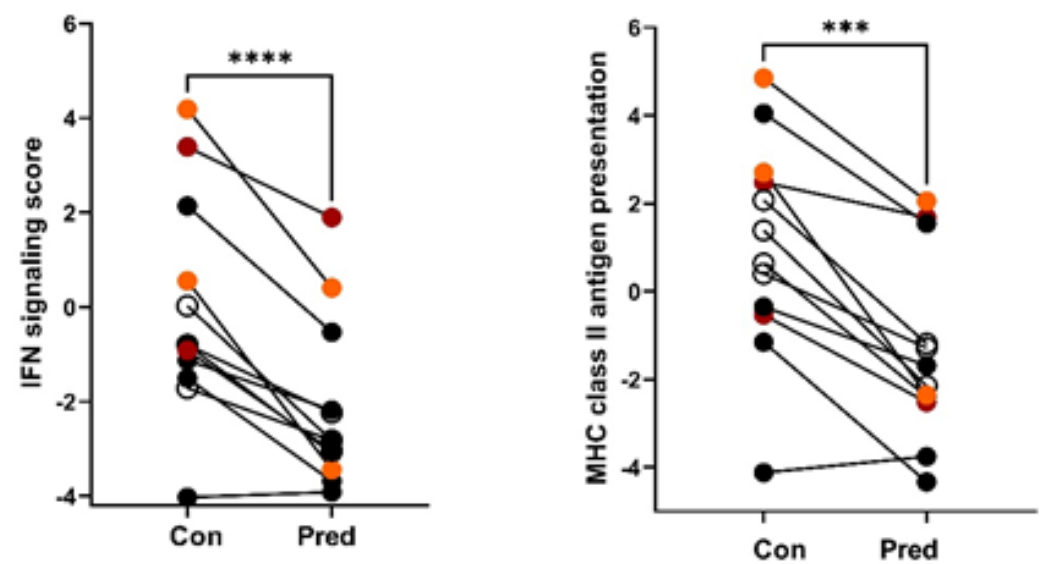

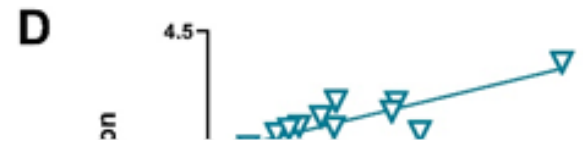

Figure 4

Transcriptomic validation of cytokine signaling pathways and prednisolone response in an independent cohort of PLHTLV-1. 
Digital transcriptomics (nCounter) was used to quantify cytokine signaling pathways in HAM/TSP disease progression, and the effect of in vitro prednisolone treatment in PBMCs from four AS, four HAM/TSP patients (including the two only incident HAM/TSP in the HOST cohort), and four age-, genderand ethnicity-matched healthy controls. (A). Ex vivo IFN signaling score was significantly higher in incident HAM/TSP as compared to healthy controls, AS, and HAM/TSP at 4-year follow-up (one sample t test). (B) Ex vivo IL-17 signaling score was significantly higher in incident HAM/TSP as compared to healthy controls and tends to decline in HAM/TSP at 4-year follow-up (one sample t test). (C) IFN signaling was homogeneously down-regulated in all clinical groups by prednisolone treatment in vitro (left panel, Wilcoxon test $p<0.0001$ ). Down-regulation was confirmed by decreased expression of the IFN$\mathrm{Y}$-regulated MHC Class II antigen presentation pathway (right panel, Wilcoxon test $p<0.001$ ). (D) Ex vivo transcriptomic IFN signaling score measured by nCounter is significantly correlated to mRNA levels of previously identified HAM/TSP biomarkers CASP5, FCGR1A, STAT1, and CXCL10 (all $\mathrm{p}<0.05$ with Bonferroni correction), but not to HTLV-1 mRNAs Hbz and Tax.

HC: Healthy controls (open circles); AS: Asymptomatics (black circles); iHAM: incident HAM/TSP (orange circles); HAM/TSP at 4-year follow-up (red circles); Con: untreated in vitro PBMCs; Pred: Prednisolonetreated PBMCs in vitro. Paired samples from iHAM patients at diagnosis and at follow-up are identified by dashed lines.

\section{Supplementary Files}

This is a list of supplementary files associated with this preprint. Click to download.

- SuppIDataAssoneetal.docx 